port and a health-resort in one borough, and which, therefore, might be taken into account in any deductions from statistics of health or mortality of their united populations.

British Association, Bristol

W. J. BLACK

\section{A Lunar Rainbow?}

THERE can be little doubt that your Australian correspondent, Mr. Lefroy (vol, xii. p. 329), has seen one of the phases of an Aurora Australis. Similar appearances have been observed by me in Scotland, passing south of the zenith (and nearly through the anti-dip, as at Fremantle). Their sudden occurrence and temporary persistence are perplexing to those who bave not seen this particular display before. The first seen by myself (in I 844 , I think) was a single beam which remained in the same position during some hours; it was described by me next day in a local paper, while a well-known observer in a communication to an Edinburgh journal had taken it for a comet.

It is pleasant to see accounts of such phenomena'sent to NATURE from all parts of the world, even when the true cause has not always been apparent. It is not improbable that the magnets at Melbourne will have shown some slight disturbance about $8 \mathrm{~h}$. 30m. P.M. of May I6.

John Allen Broun

I Do not see any reason to doubt that the phenomenon seen by " J. W. N. L." in Australia, and described by him in vol. xii. p. 329 , was an aurora. I never saw one with so many arches as he mentions (eighteen or twenty), but there can be no reason for supposing so large a number to be impossible. In almost every other respect his description agrees exactly with auroras such as may occasionally be seen.

West Hendon House, Sunderland, Sept. 4

\section{The House-Fly}

I WAS somewhat interested in Mr. Cole's remarks on the housefy in NATURE (vol. xii. p. 187), and recently had an example of another of its enemies. On touching a rather small decrepit house-fly which was making its way across a sheet of paper, three minute, active animals, apparently beetles, tumbled out of it ; they were light brown in colour, and very much the shape of aphides, and about the size of the hole a medium sized pin would make when pushed through paper.

F, P.

\section{OUR ASTRONOMTCAL COLUMN}

M. LEVERRIER'S THEORY OF SATURN.-Early in the year I $874, M$. Leverrier presented to the Paris Academy of Sciences the conclusions he had drawn from the comparison of his analytical theory of the planet Jupiter with the meridian observations made at Greenwich and Paris during the long period of I 20 years, which he found to be represented thereby with all desirable precision; thus proving that the motion of Jupiter is not subject to any sensible action beyond the effects of the known planets.

The comparison of the theory of Saturn with a similar extended course of normal positions, each one based upon a yreat number of observations, has not run quite so smoothly, but, on the contrary, has presented some slight difficulties, upon which M. Leverrier makes known his opinion in a communication to the Paris Academy on the 23 rd of last month. During the thirty-two years of modern observations, 1837.69 , the differences between theory and calculation, except in two instances, remain below $0^{\circ} 2 \mathrm{~s}$. in the times of passage observed on the meridian; for the older observations of Maskelyne and Bradley, somewhat larger discordances are shown. The residuals are, however, upon the whole, very small, and a question arises, whether such quantities can be legitimately neglected, or, if not, whether their cause is to be sought in incompleteness of the analysis or in errors of the observations themselves. M. Leverrier has not been content to rest upon the first supposition, but states that he has used every effert to elucidate the source of the remaining differences. To satisfy himself and astronomers generally that there is no defect or inaccuracy of theory, M. Leverrier has taken extraordinary pains to guard against error or omission. When he found in his earlier researches a discordance between theory and observation in the case of Mercury, he was able to explain the whole by admitting an increase in the motion of the perihelion, which might be attributed to the existence of cosmical matter or the action of small bodies nearer to the sun than the planet; and again, when the comparison of theory with the observations of Mars showed differences, they were explainable by a similar assumption of increased motion of the perihelion, necessitating an increase in the mass of the earth, and consequently of the solar parallax. In the case of Saturn, the smallness of the residuals has rendered it a much more difficult matter to pronounce with confidence upon their cause. Having reviewed the whole of his analytical theory, M. Leverrier, with the view to further verify it, considering this theory as a first, though exact approximation, proceeded by methods of interpolation to reconstruct it, taking account at once of the terms of all orders. Every possible verification having been thus accumulated, he concluded that no error was to be apprehended in this direction. The comparison with the normal positions having been certified with equal care, he ascertained the effect of small changes in the masses of Jupiter and Uranus, the errors being exhibited in functions of the corrections to these masses, and the results prove that no alteration in the adopted value of either mass will destroy the residuals as a whole; if they are somewhat diminished thereby in one part of the series, it is only at the expense of increasing them in other parts. Indeed, M. Leverrier establishes one point, and a very remarkable one it will no doubt be considered, viz., that the I 20 years of meridian observations of Saturn are insufficient to afford a reliable value of the mass of Jupiter ; or, in other words, that the mass of Jupiter which has so great an importance in the elements of the solar system, is not yet determinable from the comparison of the theory of Saturn with observations. This was not the case as regards the mass of Saturn, which M. Leverrier found from his researches upon the motion of Jupiter to be $\frac{\mathrm{I}}{3529.56}$ a somewhat smaller value than that resulting from Bessel's measures of the Huygenian satellite.

Under the above circumstances, the probability that errors of observation are the cause of the remaining differences from theory is much increased, and $M$. Leverrier appears inclined to attribute these errors to the interference of the rings under their various phases, an explanation which practical men will assuredly regard with favour. Considering that at certain times the rings disappear entirely, when the planet's centre may be well observed, while at others intervening in an elliptical form, projecting shadows and occasionally rendering impossible the observation of one of the limbs, there is nothing unlikely, as M. Leverrier remarks, in an uncertainty of some tenths of a second in R.A., which would sufficiently explain all. At any rate, whatever influence the interference of the rings may have upon the observations, it is doubtless of a variable character, as well on account of the physical fact itself, as from the effect it may exercise on personal equations.

Mr. DE LA RUE'S TABLES FOR REDUCTION OF SOLAR OBSERVATIONS. - "Auxiliary Tables for determining the angles of position of the Sun's Axis and the Latitude and Longitude of the Earth referred to the Sun's equator," which have been employed in the reduction of the tenyear series of solar photograms taken at the Kew Observatory, have just been printed by Mr. De la Rue, professedly for private circulation, though, as they have been imposed in the size and type of the "Philosophical Trans- 